Results Over the study period, 1533 female patients were tested for TV using the rapid test, of which 539 (35.2\%) were Black, with a median age of 28 years (range $6-82$ years). The TV positivity rate based on the rapid test was $5.6 \%(86 / 1533)$, compared to $3.2 \%$ (54/1681) among those tested with WM. Among 77 unique female patients with TV infection, 467 (34.0\%) were Black and had a median age of 28 years. Nearly half of infected women presented with a chief complaint of abdominal pain (49\%); vaginal discharge was only reported by $10 \%$. Among infected women, $55 \%$ had concomitant bacterial vaginosis (BV), and 16\% were co-infected with gonorrhoea and/or chlamydia. Most infected women (84\%) were prescribed metronidazole during the same ER visit.

Conclusions. The OSOM ${ }^{\circledast}$ Trichomonas Rapid Test resulted in a $60 \%$ increase in TV detection among women compared to WM, and the majority received appropriate TV therapy. Women identified with TV infection in an ER setting were primarily co-infected with BV and other STIs.

\section{P2.078 DRUG RESISTANCE OF MYCOBACTERIUM TUBERCULOSIS AT PATIENTS WITH COMBINATION OF TUBERCULOSIS AND THE HIV INFECTION}

doi:10.1136/sextrans-2013-051184.0343

'G S Balasanyants, 'E A Torkatjuk, ${ }^{2} \mathrm{~T}$ Y Suprun. 'St.-Petersburg scientific research institute Phthisiopulmonology, St.-Petersburg, Russian Federation; ${ }^{2}$ Tb hospital No 2 St.-Petersburg, St.-Petersburg, Russian Federation

Purpose To estimate of drug resistances of $(\mathrm{MBT})$ at patients with combination of tuberculosis and the HIV (TB+HIV).

Materials and Methods Patients with confection treated in tubercular hospital №2 for the period of 2005-2011

Results For the specified period the among patients with TB+HIV the share of low immune status (less than $200 \mathrm{cell} / \mathrm{mkl}$ ), causing severe TB has increased from $32.5 \%$ up to $60.4 \%$. It was accompanied by growth generalised forms of $\mathrm{TB}$ from $30.1 \%$ up to $56.5 \%$.

MBT excreting it has been identified at $67.7 \% \mathrm{~TB}+\mathrm{HIV}$ cases, and in $53.2 \%$ cases baccilar TB have been revealed by culture. From them only at $19.9 \%$ of MBT were sensitive to antitubercular drugs, at others tests identified presence of resistance of various prevalence. Resistance to streptomycin (73.2\%) and izoniasid $73.4 \%$ was more often, it is a little bit less - to rifampicin - than $63.3 \%$, and ethambutol $-41.2 \%$. Among second line TB drugs the greatest resistance has been fixed to canamycin $-36.7 \%$, to other drugs of this group it did not exceed $20 \%$.

The proportion of patients with multi drug resistant MBT has determined in an interval of $80.6 \%$ of $-65.3 \%$; among patients with new cases of TB it was on the average $73.3 \%$. Extremely drug resistant $\mathrm{MBT}$ (XDR) has registered among for the new cases within $7.9 \%-14.8 \%$. Patients with XDR had expressed immune suppression and progressing of tuberculosis with development generalised forms even at use of ART. Lethal outcomes at them made $56.9 \%$.

Conclusion wide circulation of multi and extremely drug resistant MBT at patients with TB+HIV makes inefficient treatment at prescription of $\mathrm{f}$ standard TB schemes. Besides for these patients use of methods of fast identification of drug resistance is necessary.

\section{P2.079 WITHDRAWN BY AUTHOR}

\section{P2.080 DIVERSITY OF NEISSERIA GONORRHOEAE ANTIMICROBIAL SUSCEPTIBILITY TESTING METHODOLOGIES IN THE UNITED KINGDOM}

doi:10.1136/sextrans-2013-051184.0344

${ }^{1}$ A Jain, ${ }^{2 T}$ Planche, ${ }^{1} \mathrm{C}$ Ison. 'Sexually Transmitted Bacteria Reference Unit, Health Protection Agency, London, UK; ${ }^{2}$ St George's Healthcare NHS Trust, London, UK
Background Appropriate and timely treatment for Neisseria gonorrhoeae infection is an essential clinical and public health action. Antimicrobial susceptibility testing (AST) predicts therapeutic failure and guides selection of appropriate treatment.

Increasing antimicrobial resistance in $N$. gonorrhoeae prompted the publication of the global action plan by the World Health Organisation to control its spread. This document highlighted the lack of or use of different methodologies for AST making the inter-laboratories and international comparisons and monitoring difficult.

Aims The aim of this study was to explore whether laboratories offered AST for $N$. gonorrhoeae and which methodologies were being used to detect resistance particularly to current recommended treatment.

Methods A web based survey with 23 questions regarding AST was developed and rolled out to the members of British Society for Microbiology Technology and UK Standards for Microbiology Investigations from November 2012 to January 2013.

Results There were 327 responses from 118 laboratories from across the UK. After excluding duplicate and empty responses, 206 responses were analysed.

196 respondents (95\%) conducted AST for $N$. gonorrhoeae with $46 \%$ performing this daily. 147 respondents (75\%) used British Society of Antimicrobial Chemotherapy method and 8\% used European Committee on Antimicrobial Susceptibility Testing breakpoints. $80 \%$ respondents always tested penicillin susceptibility, $71 \%$ always tested ceftriaxone and 55\% always tested azithromycin. The most common methods used were disc diffusion (73\% respondents) and E-test (48\% respondents). 26\% respondents did not archive isolates with potentially decreased susceptibility to cephalosporins and $19 \%$ did not use control strains for AST.

Conclusion This study highlights the diversity in approach to AST by different laboratories across the UK. Ceftriaxone and azithromycin, the antibiotics of choice for uncomplicated anogenital infections, were not consistently tested. AST is the basis for detecting resistance and modifying therapy accordingly and a consistent approach is required for both patient treatment and surveillance.

\section{P2.081 ANTIMICROBIAL SUSCEPTIBILITIES OF NEISSERIA GONORRHOEAE STRAINS FROM MALE URETHRITIS IN JAPAN -THE FIRST NATIONAL SURVEILLANCE}

doi:10.1136/sextrans-2013-051184.0345

1.2R Hamasuna, ${ }^{2} \mathrm{M}$ Yasuda, ${ }^{2} \mathrm{~K}$ Ishikawa, ${ }^{2} \mathrm{~S}$ Uehara, ${ }^{2} \mathrm{~S}$ Takahashi, ${ }^{2} \mathrm{H}$ Hayami, ${ }^{2} \mathrm{~S}$ Yamamoto, ${ }^{2} \mathrm{~T}$ Matsumoto, ${ }^{3} \mathrm{H}$ Hanaki, ${ }^{2} \mathrm{~J}$ Kadota. ${ }^{1}$ Department of Urology, University of Occupational and Environmental Health, Japan, Kitakyushu, Japan; ${ }^{2}$ The members of Urogenital Sub-committee and the surveillance committee of Japanese Society of Chemotherapy (JSC), Japanese Association for Infectious Diseases (JAID) and Japanese Society for Clinical Microbiology (JSCM), Tokyo, Japan; ${ }^{2}$ Research Center for Anti-Infectious Drugs, Kitasato University, Tokyo, Japan

Background Neisseria gonorhoeae is one of the most important pathogens causing sexually transmitted infection. Resistant $N$. gonorrhoeae trains against several antimicrobials are increasing worldwide.

Purpose In this study, the trends of antimicrobial susceptibilities among $N$. gonorrhoeae strains isolated from male patients with urethritis were investigated as the first Japanese national surveillance, which was conducted by the surveillance committee of three Japanese societies as Japanese Society of Chemotherapy, Japanese Association of Infectious Diseases and Japanese Society of Clinical Microbiology.

Methods The targets were male patients older than 16 years with urethral discharge and symptoms of urethritis. The patients were diagnosed with gonococcal urethritis by a clinician at 51 participating facilities. The period of specimen collection was between April 2009 and October 2010. 